\title{
Assessing the effective use of mosquito nets in the prevention of malaria in some parts of Mezam division, Northwest Region Cameroon
}

Ngum Helen Ntonifor ${ }^{*}$ and Serophine Veyufambom²

\begin{abstract}
Background: In Cameroon, malaria continues to be endemic and the first major cause of morbidity and mortality among the most vulnerable groups_children under 5 years of age, pregnant women, people living with HIV/AIDS and the poor. The use of long-lasting insecticide-treated bed nets (LLINs) is one of the recommended measures to prevent malaria. The present study was aimed at accessing the acceptability and effective use of LLINs on the prevalence of malaria in PMI Nkwen, Bambui and its environs.
\end{abstract}

Methods: Hospital-based diagnosis consisted of 476 blood samples that were screened using the rapid diagnostic kits to determine the prevalence of malaria among users and non-users of LLINs. A structured questionnaire was also administered to pregnant women and children less than 5 years of age (476 hospital-based and 350 from the community) which consisted of demographic information, availability, accessibility affordability, acceptability, effective use and problems encountered with the use of LLINs.

Results: Result obtained showed that out of the 476 hospital-based patients, 29 tested positive for malaria giving an overall prevalence of $6.09 \%$. Equally, results of the questionnaire showed that $743(89.9 \%)$ of the respondents owned LLINs with up to 649 (87.3\%) having been given to them free-of-charge, and that 578 (77.8\%) were using their LLINs to sleep, even though $18.2 \%$ of the respondents used their LLINs for other purposes, such as fishing, nursing seeds and footfall nets. Malaria was minimal among users of LLINs than non-users and the results were significant at $P \leq 0.05$. Also $71.9 \%$ of the respondents said that their nets were in good condition while $52.2 \%$ of them said the major problem with the usage of LLINs was heat and the feeling of suffocation.

Conclusion: These results indicate that LLINs have significantly reduced the prevalence of malaria among the studied population, and so the government should not relent its efforts in the distribution of these nets especially to the vulnerable groups in order eliminate malaria and other mosquito-borne diseases. Utilization of LLINs needs to be encouraged to match ownership, while free distribution of ITNs to vulnerable groups needs to be continuous and consistent.

Keywords: LLINs, Malaria, Prevention, Pregnancy and children 0-5 years, Cameroon

\section{Background}

Malaria remains the main threat to public health despite decades of control efforts made. It is a devastating disease that threatens productivity and economy of endemic countries [1]. There were over 214 million new cases of

\footnotetext{
*Correspondence: ngumnto@yahoo.com

1 Department of Biological Sciences, University of Bamenda, North West Region, Bambili, Bamenda, Cameroon

Full list of author information is available at the end of the article
}

malaria worldwide in 2015 and approximately 438,000 malaria deaths [2]. It constitutes over $10 \%$ of Africa's overall disease burden, accounting for $40 \%$ of public health expenditure, $30-50 \%$ of in-patient hospital admissions and up to $50 \%$ of out-patient visits in endemic areas [3].

Over the last century, efforts have been made to control malaria. Among the new advances in the control of malaria is the use of insecticide-treated nets (ITNs), now 
mostly long-lasting insecticide-treated nets (LLINs). ITNS are known to kill mosquitoes and have proven repellent properties that reduce the number of mosquitoes that enter the house [3]. They are estimated to be twice as effective as untreated nets [4] and offer greater than $70 \%$ protection compared with no nets [5].

In the past decade, malaria incidence has fallen by at least 50 percent in one-third of the countries where the disease is endemic. These gains have been made through a combination of interventions, including timely diagnosis and treatment using reliable tests and anti-malarial drugs; indoor spraying with safe insecticides; and the use of LLINS to protect people from mosquito bites at night.

Mosquito net ownership is far from universal use despite the aforementioned gains. Ownership rates remain low in many malarious regions or amongst particular groups in malarious regions. Furthermore, mosquito net ownership in itself is not synonymous with utilization. Also ownership is not the only obstacle to achieving the reduction in malaria morbidity and mortality associated with ITN use. Rather, individuals who own (or who have available) mosquito nets must use them in order for the potential health impact to be fully realized [6]. The main strategies for malaria prevention in Cameroon are intermittent preventive treatment (IPT) for pregnant women and vector control through the use of ITNs especially for pregnant women and under-five children [7]. ITNs distributed free-of-charge have been in existence in households in Cameroon since 2003 following the Abuja Declaration in 2000. ITNs are also being distributed in the country free-of-charge to pregnant women during antenatal clinics (ANC) while the rest of the community members obtain their own ITNs from the regional treatment units (RTU) and community treatment units (CTUs) where ITNs are re-impregnated with insecticides after regular intervals of 6 months by community relay agents (CRA), who have been trained to carry out this exercise [2]. While challenges to increasing ITN ownership may diminish as a result of the expansion of large-scale distribution efforts, ITN impact on transmission will be minimized if they are not properly and consistently used, especially among populations vulnerable to increased malaria morbidity and mortality, such as children and pregnant [7].

\section{Methods}

\section{Study area}

The study was carried out in PMI Nkwen, Bamenda (Urban setting) and in Bambui (Rural setting), respectively. Bamenda is located in the North West region of Cameroon. It has a population of high-income earners comprising mostly of civil servants and business men. The population density is high while housing facilities are poor. Bambui is located in the Eastern part of Northwest Region of Cameroon. It is the headquarter of Tubah sub-division, located at $6^{\circ} 3^{\prime} 0^{\prime \prime}$ North, and $10^{\circ} 14^{\prime} 0^{\prime \prime}$ East and about $1350 \mathrm{~m}$ above sea level. It has a population of about 50,000 people, with very fertile soils and the majority of its inhabitants are farmers, petty traders and students. These two health areas have common characteristics, such as two seasons (rainy and dry seasons), poor town planning and construction with each room having only one door and one window. Empty plots serve as refuse disposal sites, and defecation places, as most of the bungalows have inadequate or no toilet facilities and no water. In these conditions of very low environmental sanitation and poor personal hygiene, infective diseases abound.

\section{Sample size and study population}

The study population consisted of all patients (pregnant women and children) in PMI Nkwen and Bambui district health centre and respondents in these communities who gave their consent to participate in the study were enrolled. Pregnant women and children included those with or without LLINs.

\section{Research design}

A cross sectional survey was designed to include all children from $0-5$ years and pregnant women. The respondents were given questionnaires on their knowledge on the availability, acceptability, effective use of LLINs and the problems faced during the usage.

\section{Parasitological diagnosis}

Thick and thin blood films were prepared, stained with $5 \%$ Giemsa for $30 \mathrm{~min}$ and examined under the $\times 100$ (oil immersion) objective of a $\mathrm{UNICO}^{\circledR}$ light microscope for the identification of the malaria parasite. Slides were declared negative if no asexual parasites or gametocytes were found after examining 100 high-power fields. For each of the positive slides, parasite density per $\mu \mathrm{l}$ of blood was determined in thick smear on the basis of the number of parasites per 200 leucocytes with reference to participants' absolute WBC counts [8].

\section{Statistical analysis}

All data collected were entered into SPSS (Statistical Package for the Social Sciences) version 19 (SPSS, Inc, Chicago, IL, USA) for analyses. The frequency of malaria attacks were log transformed before analysis. Associations between the use of LLINs, condition of LLINs, age, how often the nets were washed, education on the use of LLINs, level of education, and malaria prevalence were evaluated using Pearson Chi Square $\left(x^{2}\right)$ test. Differences in group means were compared using ANOVA, Student's 
$t$ test, Mann-Whitney U or Kruskal-Wallis test. Multinomial logistic regression model was used to determine risk factors associated with using LLINs and malaria. Statistical significance was set at $\mathrm{P}<0.05$.

\section{Results}

\section{Characteristics of the study population}

A total of 826 respondents were recruited for the study out of which 476 (57.62\%) were from the hospital and $350(42.38 \%)$ were from the community. Out of this lot, 495 (59.9\%) were children aged $0-5$ years old while 331 (40.1\%) were pregnant women aged 15-48 years. Respondents that were based in PMI and its environs were $459(55.57 \%)$ while those that were from Bambui and its environs were 367 (44.43\%). Out of the 476 respondents that were hospital-based, 279 (58.6\%) of children and 146 (30.6\%) of pregnant women had nets while $40(6.4 \%)$ children and $11(4.4 \%)$ women did not have.

\section{Overall prevalence of malaria in the study population} Out of the 476 hospital-based patients, 29 tested positive for malaria giving an overall prevalence of $6.09 \%$. The prevalence of malaria in children $6.7 \%(22 / 319)$ was however higher than that of pregnant women $4.46 \%$ $(7 / 157)$ even though the difference was not statistically significant at $\mathrm{P} \leq 0.05$. With regards to children, $2.87 \%$ $(8 / 279)$ who were positive for malaria were using LLINs while $35 \%(14 / 40)$ were not using LLINs. On the other hand, the seven pregnant women who had malaria were not using LLINs (Table 1).

\section{Associated demographic data with usage, and like to use LLINs}

Respondents were examined in relation to their educational level and the usage of LLINs and from Table 2, 315 $(42.3 \%)$ of respondents from Bambui were using LLINs, compared to 430 (57.7 \%) from PMI Nkwen. Equally 457 $(61.3 \%)$ children aged $0-5$ years and 288 (38.7\%) women aged 15-48 years old were using their bed nets. With regards to those who liked using bed nets, 311 (84.7\%) from Bambui liked using LLINs and 443 (96.5\%) from PMI equally liked using them (Table 2).

\section{Distribution of study population in relation to the frequency of malaria treatment}

Out of the 826 respondents, $272(32.93 \%)$ said they had not been treated for malaria for the past 1 year, $67(8.11 \%)$ said they were treated monthly while 202 $(24.46 \%)$ said they were treated yearly (Fig. 1$)$. According to the respondents using LLINs, 16 (2.34\%) said that they were treated monthly, $43(7.19 \%)$ almost every 6 months, $133(22.24 \%)$ yearly, while for those who were not using LLINs, $24(16.33 \%)$ said they were treated monthly, 41 $(27.87 \%)$ six monthly and $43(29.25 \%)$ yearly. However, $81(9.8 \%)$ did not respond to the questions (see details on Table 3).

Analysis on how often the respondents were treated for malaria versus the condition of their LLINs was done, with the following results; for those whose net was in good condition, none was treated monthly, 15 (3.5\%) 6 monthly, 90 (21.9\%)yearly, while 181 (44.1\%) said they were not treated at all. For those whose LLINs were in acceptable condition, 4 (3.9\%) were treated monthly, 10 (9.7 \%) 6 monthly, and 18 (17.5\%) yearly, while those with LLINs in poor condition $12(27.9 \%)$ were treated monthly, 18 (41.9 \%) 6 monthly, and 7 (16.3\%) yearly (Table 3).

\section{Accessibility and acceptability of LLINs among respondents} The accessibility of LLINs is displayed on Table 4. Out of the 743 respondents that owned mosquito nets, 94 (12.7 \%) bought them, while 649 (87.3\%) had been given them free-of-charge by the government. Equally 596 (72.2 \%) of the respondents said they could get LLINs from the hospital, while 78 (4.8\%) did not know where to get LLINs from. In their opinion on the cost of LLINs, $226(27.4 \%)$ said it was cheap, $128(15.5 \%)$ said it was average, while $21(2.1 \%)$ said it was expensive and 451 $(54.6 \%)$ did not know at all and the results were statistically significant at $\mathrm{P} \leq 0.05$. With regards to the acceptability of LLINs; 754 (91.3 \%) respondents liked using mosquito nets against malaria, while $72(8.7 \%)$ did not. Out of the 826 respondents, 598 (95.2\%) were using their LLINs and 30 (4.8\%) were not using them, while $198(24 \%)$ did not answer. Out of the lot that was using LLINs, 578 (96.7 \%) were actually using nets to sleep

Table 1 Prevalence of malaria in relation to the usage of LLINs amongst children and pregnant women

\begin{tabular}{|c|c|c|c|c|c|c|c|c|}
\hline & \multirow{2}{*}{$\begin{array}{l}\text { Number } \\
\text { of patients }\end{array}$} & \multirow{2}{*}{$\begin{array}{l}\text { Number of patients } \\
\text { using LLINs }\end{array}$} & \multicolumn{2}{|c|}{ Number of infected cases } & \multirow[t]{2}{*}{ Total } & \multicolumn{2}{|c|}{ Prevalence of malaria (\%) } & \multirow[t]{2}{*}{$P$ value } \\
\hline & & & $\begin{array}{l}\text { Using } \\
\text { LLINs }\end{array}$ & $\begin{array}{l}\text { Without } \\
\text { LLINs }\end{array}$ & & $\begin{array}{l}\text { Using } \\
\text { LLINs }\end{array}$ & $\begin{array}{l}\text { Without } \\
\text { LLINs }\end{array}$ & \\
\hline Children & 319 & 279 & 8 & 14 & 22 & 2.87 & 35 & 0.00 \\
\hline Pregnant women & 157 & 147 & 0 & 7 & 7 & 0 & 4.46 & 0.01 \\
\hline Total & 476 & 426 & 8 & 21 & 29 & 2.87 & 39.46 & \\
\hline
\end{tabular}


Table 2 Associated demographic information and usage of LLINs

\begin{tabular}{|c|c|c|c|c|c|c|c|c|c|}
\hline & & \multicolumn{4}{|c|}{ Usage of LLINs } & \multicolumn{4}{|c|}{ Like to use LLINs } \\
\hline & & Yes N (\%) & No N (\%) & Total N (\%) & $x^{2}$ & Yes N (\%) & No N (\%) & Total N (\%) & $x^{2}$ \\
\hline \multirow[t]{3}{*}{ Village } & Bambui & $233(73.9)$ & $82(26.0)$ & $315(42.3)$ & 1.6 & 311 (84.7) & $56(15.3)$ & $367(44.4)$ & 34.8 \\
\hline & PM I Nkwen & $365(84.9)$ & $65(15.1)$ & $430(57.7)$ & 9.9 & $443(96.5)$ & $16(3.4)$ & $459(55.6)$ & 23.1 \\
\hline & Total & $598(80.3)$ & $147(19.7)$ & $745(100)$ & & $754(91.3)$ & $72(8.7)$ & $826(100)$ & \\
\hline \multirow[t]{3}{*}{ Age } & $0-5$ & $356(77.9)$ & $101(22.1)$ & $457(61.3)$ & 6.78 & 478 (96.6) & $21(4.2)$ & $495(59.9)$ & 46.5 \\
\hline & $15-48$ & $242(84.0)$ & $46(15.9)$ & $288(38.7)$ & 14.3 & $280(84.6)$ & $51(15.4)$ & $331(40.1)$ & 7.02 \\
\hline & Total & $598(80.3)$ & $147(19.7)$ & $745(100)$ & & 754 (91.3) & $72(8.7)$ & $826(100)$ & \\
\hline \multirow[t]{5}{*}{ Level of education } & No schooling & $108(81.8)$ & $24(18.2)$ & $132(\%)$ & 3.35 & $127(88.2)$ & $17(11.8)$ & $144(17.4)$ & 0.09 \\
\hline & Primary & $144(62.1)$ & $88(37.9)$ & $232(31.1)$ & 0.093 & $232(96.6)$ & $8(3.3)$ & $240(29.1)$ & 0.41 \\
\hline & Secondary (1st cycle) & $102(97.1)$ & $3(2.9)$ & $105(14.1)$ & 6.73 & $102(94.4)$ & $6(5.6)$ & $108(13.1)$ & 12.7 \\
\hline & Secondary (2nd cycle) & $134(85.9)$ & $22(14.1)$ & $156(20.9)$ & 12.39 & $138(79.3)$ & $36(20.7)$ & $174(21.1)$ & 9.68 \\
\hline & University & $110(91.7)$ & $10(8.3)$ & $120(16.1)$ & 5.63 & 155 (96.9) & $5(3.1)$ & $160(19.4)$ & 8.60 \\
\hline Total & Total & $598(80.3)$ & $120(16.1)$ & $745(100)$ & & 754 (91.3) & $72(8.7)$ & $826(100)$ & \\
\hline
\end{tabular}

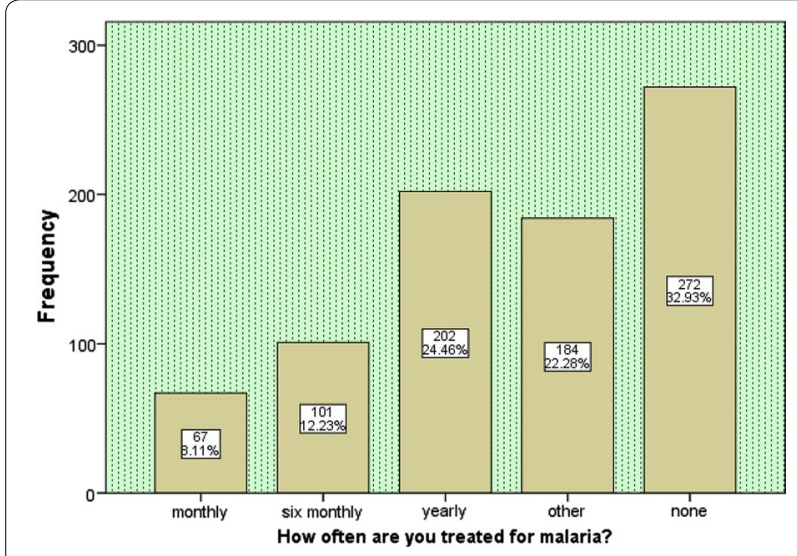

Fig. 1 Frequency of malaria treatment among respondents

under while $20(3.4 \%)$ were for other activities, such as football nets, nursing seeds and wall coverings (Fig. 2).

\section{Effective use of LLINs among respondents}

The effective usage of LLINs varied among respondents as shown on Table 5. Out of the 826 respondents, 261 (50.2\%) said they use to pull down their LLINs before they got to bed, 158 (30.4\%) fitted them well to the bed before sleeping, $59(11.4 \%)$ put them permanently over their beds and $42(8.1 \%)$ of them used nets which were free from holes and could correctly fit the nets over their beds within the right hours. In addition, 374 (71.9 \%) of the total population had mosquito nets which were of good conditions, while 306 (37.0\%) did not respond to the question. These results were significant at $\mathrm{P} \leq 0.049$.

The condition and longevity of LLINs as indicated by the respondents was also analysed. Results showed that $4(0.09 \%)$ respondents had LLINs that were in good conditions and older than 4 years, 36 (8.8\%) were 3 years old, while $240(58.5 \%)$ were 1 year old (Table 5). 410 (73.7 \%) of the respondents said their nets were in very good condition, while $43(7.7 \%)$ respondents said that theirs were in poor conditions.

\section{Problems encountered by respondents using LLINs}

Some of the problems surrounding the use of LLINs were analysed. $517(87.9 \%)$ of the respondents said it was easy putting on LLINs, while 17 (12.1\%) said it was not easy. 307 (52.2 \%) respondents said the nets were making them feel hot, while 111 (18.9\%) said they were tired and lazy to put the nets on in the evenings. $91.8 \%$ of the respondents were comfortable with the use of mosquito nets, while only $8.2 \%$ were not.

\section{Public health awareness on using LLINs in the prevention of malaria}

Out of the 826 respondents recruited for the study, only $588(71.2 \%)$ responded to the public awareness section out of which $527(89.6 \%)$ said they had been taught on how to use mosquito nets, while for education on the use of mosquito nets in the prevention of malaria, 760 (95.8\%) of the respondents agreed that they had been educated.

\section{Discussion}

The study revealed an overall prevalence of malaria of $6.09 \%$. These results are in line with those of Sohail et al. [9], who got a prevalence of 5.4 in pregnant women, and to previous reports of $7.9 \%$ by Leke et al. [10], and Mbu et al. [11], who reported $6.6 \%$ of malaria cases among women at routine ANC clinic visits. However, malaria prevalence was less than that reported for out-patient 
Table 3 Frequency of malaria treatment

\begin{tabular}{|c|c|c|c|c|c|c|c|c|c|}
\hline & & \multicolumn{5}{|c|}{ Frequency of malaria treatment } & \multirow[t]{2}{*}{ Total N (\%) } & \multirow[t]{2}{*}{$\mathrm{X}^{2}$} & \multirow[t]{2}{*}{$P$ value } \\
\hline & & Monthly N (\%) & Six monthly N (\%) & Yearly N (\%) & Other N (\%) & None N (\%) & & & \\
\hline \multirow[t]{3}{*}{ Are using LLINs } & Yes & $16(2.3)$ & $43(7.2)$ & $133(22.2)$ & $148(24.8)$ & $258(43.1)$ & $598(80.3)$ & 70.3 & 0.00 \\
\hline & No & $24(16.3)$ & $41(27.8)$ & $43(29.3)$ & $28(18.1)$ & $11(7.48)$ & $147(19.7)$ & 36.6 & 0.00 \\
\hline & Total & $40(5.4)$ & $84(11.3)$ & $176(23.6)$ & $176(23.6)$ & $269(36.1)$ & $745(100)$ & & \\
\hline \multirow[t]{4}{*}{ Condition of LLINs } & Very good & $0(\%)$ & $15(3.7)$ & $90(21.9)$ & $124(30.2)$ & $181(44.1)$ & $410(73.7)$ & 42.1 & 0.00 \\
\hline & Ok & $4(3.9)$ & $10(9.7)$ & $18(17.5)$ & $26(25.2)$ & $45(43.6)$ & $103(18.5)$ & 6.41 & 0.09 \\
\hline & Poor & $12(27.9)$ & $18(41.9)$ & $7(16.3)$ & $4(9.3)$ & $2(4.7)$ & $43(7.7)$ & 2.88 & 0.41 \\
\hline & Total & $16(2.9)$ & $43(7.7)$ & $115(20.7)$ & $152(27.3)$ & $230(41.4)$ & $556(100)$ & & \\
\hline \multirow{5}{*}{$\begin{array}{l}\text { How often LLINs is } \\
\text { used }\end{array}$} & Everyday & 0 & $15(3.8)$ & $63(16.1)$ & $106(27.1)$ & $207(52.9)$ & $391(66.5)$ & 57.9 & 0.00 \\
\hline & When sick & $4(16)$ & $9(36)$ & $7(28)$ & $3(12)$ & $2(8)$ & $25(4.3)$ & 15.9 & 0.00 \\
\hline & When at home & $3(2.7)$ & $12(10.7)$ & 47 (41.9) & $23(20.5)$ & $27(24.1)$ & $112(19.1)$ & 19.8 & 0.00 \\
\hline & Not often & $10(16.7)$ & $14(23.3)$ & $19(31.7)$ & $9(15)$ & $8(13.3)$ & $60(10.2)$ & 6.50 & 0.04 \\
\hline & Total & $17(2.9)$ & $50(8.5)$ & $136(23.1)$ & $141(23.9)$ & $244(41.5)$ & $588(100)$ & & \\
\hline \multirow[t]{2}{*}{ Age } & $0-5$ & $51(10.3)$ & $58(11.7)$ & 77 (15.6) & $115(23.2)$ & $194(39.1)$ & 495 (59.9) & 28.9 & 0.02 \\
\hline & $15-48$ & $18(5.4)$ & $50(15.1)$ & $123(37.2)$ & $69(20.8)$ & $71(21.5)$ & $331(40.1)$ & 7.76 & 0.00 \\
\hline Total & & $69(8.4)$ & $108(13)$ & $200(24.1)$ & $184(22.3)$ & $265(32.1)$ & $826(100)$ & & \\
\hline
\end{tabular}

Table 4 Accessibility of LLINs among respondents

\begin{tabular}{llr}
\hline Source of net & N (\%) & Total (\%) \\
\hline Bought & $94(12.7)$ & $94(12.7)$ \\
Freely given & $649(87.3)$ & $649(87.3)$ \\
Total & $743(100)$ & $743(100)$ \\
$X^{2}$ & 14.4 & \\
$p$ value & 0.000 & \\
\hline
\end{tabular}

visits by Ndo et al. [12], Egbuche et al. [3], and AnchangKimbi et al. [13]. The low prevalence of malaria in this study was probably due to the fact that most of the respondents were pregnant women and children who had bed nets and who were well educated during antenatal visits on the importance of using these nets. Added to this was the fact that the field studies were carried out during the dry season when malaria transmission is at

Table 5 Effective usage of mosquito nets and the condition of these LLINs

\begin{tabular}{|c|c|c|c|c|c|c|c|}
\hline & & \multicolumn{4}{|l|}{ Condition of LLINs } & \multirow[t]{2}{*}{$x^{2}$} & \multirow[t]{2}{*}{$P$ value } \\
\hline & & Very good N (\%) & Ok N (\%) & Poor N (\%) & Total N (\%) & & \\
\hline \multirow[t]{5}{*}{ Effective use means } & Pulling net down over bed before sleeping & $179(68.6)$ & $52(19.9)$ & $30(11.5)$ & $261(50.2)$ & 6.28 & 0.39 \\
\hline & Net well fitted on bed in the evening & $124(78.5)$ & $26(16.5)$ & $8(5.1)$ & $158(30.4)$ & 6.06 & 0.108 \\
\hline & Net putt permanently over the bed & $39(66.1)$ & $15(25.4)$ & $5(8.5)$ & $59(11.4)$ & 14.77 & 0.022 \\
\hline & $\begin{array}{l}\text { Net free from holes and fitted correctly over bed } \\
\text { within the right hours }\end{array}$ & $32(76.2)$ & $10(23.8)$ & $0(0 \%)$ & $42(8.1)$ & 2.25 & 0.324 \\
\hline & Total & $374(71.9)$ & $103(19.8)$ & $43(8.3)$ & $520(93.5)$ & & \\
\hline \multirow[t]{5}{*}{ Age of LLINs (years) } & 1 & $240(58.5)$ & $16(15.5)$ & $0(0 \%)$ & $256(46.0)$ & 17.1 & 0.009 \\
\hline & 2 & $130(31.7)$ & $26(25.2)$ & $5(11.6)$ & 161 (28.9) & 51.35 & 0.000 \\
\hline & 3 & $36(8.1)$ & 35 (33.9) & $7(16.3)$ & $78(14)$ & 100.5 & 0.000 \\
\hline & 4 & $4(0.01)$ & $26(25.2)$ & $31(72.1)$ & $61(10.9)$ & 37.69 & 0.000 \\
\hline & Total & $410(73.7)$ & $103(18.5)$ & $43(7.7)$ & $556(100)$ & & \\
\hline \multirow{4}{*}{$\begin{array}{l}\text { How often LLINs is } \\
\text { washed }\end{array}$} & None & 318 (91.1) & $28(8.0)$ & $3(0.85)$ & $349(62.8)$ & 10.79 & 0.095 \\
\hline & Once & $66(67.4)$ & $28(28.5)$ & $4(4.1)$ & 98 (17.6) & 9.97 & 0.126 \\
\hline & Twice & $23(29.5)$ & $43(55.1)$ & 10 (12.8) & $78(14)$ & 20.81 & 0.002 \\
\hline & Often & $3(9.1)$ & 4 (12.1) & $26(78.8)$ & $33(5.9)$ & 18.07 & 0.006 \\
\hline Total & & $410(73.7)$ & $103(18.5)$ & $43(7.7)$ & $556(100)$ & & \\
\hline
\end{tabular}




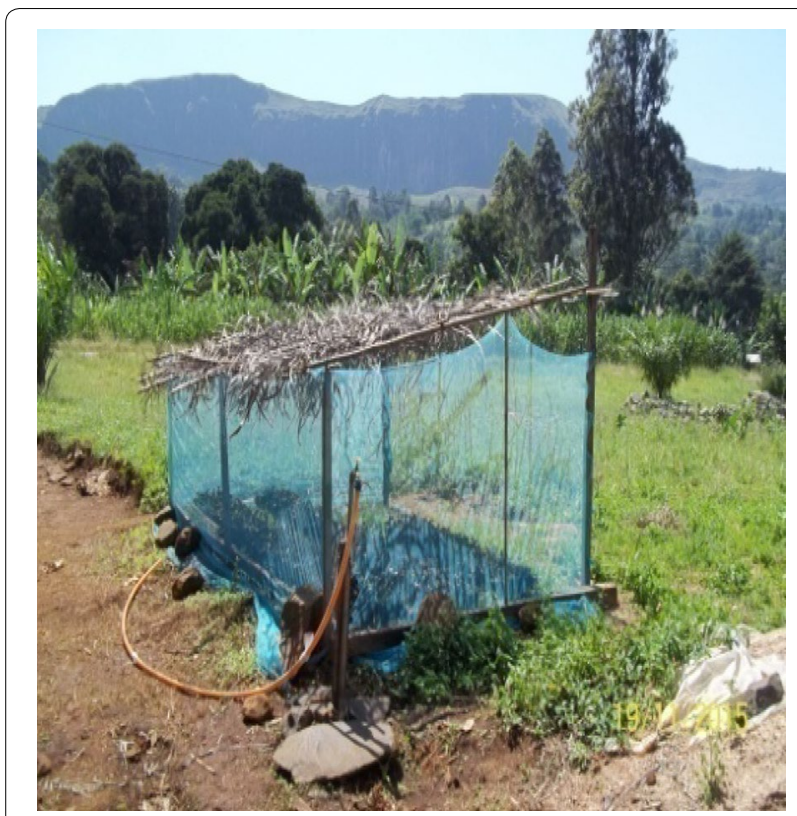

Fig. 2 Net used for nursing seeds

its minimum. Plasmodium infections usually increased during the rainy season [14]. The use of LLINs has significantly reduced malaria infection among users as for children. In the present study, it was observed that malaria prevalence in children who were not using LLINs was very high (35\%) compared to those who were using LLINs $(2.87 \%)$. The same trend was observed for the pregnant women. This confirms the report of the World Health Organization (WHO) [15], and Ergot et al. [16] on the use of LLINs as a means to reduce the lethal impact of malaria. Malaria occurred at higher prevalence among non-users and this is in line with the works of Ergot et al. [16]. The prevalence of malaria in children was higher than that of pregnant women and this was probably because of the absence of protective immunity in the children and the fact that they could easily roll out of their LLINs at night without their guardians/parents noticing. The respondents whose LLINs were in good conditions were not treated monthly of malaria and 181 $(44.1 \%)$ were not treated at all within this period. This shows that the condition of LLINs plays an important role in the prevention of mosquito bites. In addition, respondents who used their LLINs everyday were not treated monthly for malaria and up to 207 (52.9\%) of them were not treated within this period.

The availability of LLINs was very high, most of which had been given free-of-charge by the government. This result is not consistent with the findings of Pulford et al. [6], who explained that ownership rates remained low in many malarious regions or amongst particular groups in malarious regions. Also Kimbi et al. [17] reported that, only $47 \%$ of households interviewed owned at least one mosquito bed net in Buea Health District-Cameroon. LLINs were highly available in these localities because of the effort of the Cameroon government in collaboration with some funding bodies like UN, WHO and UNICEF to achieve the Sixth MDGs by so doing. LLINs are given to all pregnant women during the first antenatal visit to the health centre. In this light one of the major recommendations for malaria control under the Roll Back Malaria initiative in Cameroon since 2013 was free distribution of LLINs to pregnant women and children under 5 years of age [12]. Furthermore, residents of the North West, Centre and East Regions of Cameroon continue to receive free LLINs from other partners, such as PLAN Cameroon (a non-governmental organization).

LLINs were equally accessible to the people as $90.6 \%$ of the respondents knew where to get the nets from. In addition to this, most of the respondents did not know how much LLINs could cost probably because they were used to free donations. This confirms the WHO measures [18] to reduce malaria morbidity and mortality through large-scale programmes of distribution of free or highly subsidized LLINs, which lastly took place in October 2015 in the North West and South West Regions of Cameroon. Despite the fact that many did not know the cost of LLINs, the few that knew were of the opinion that the nets were cheap making them affordable to those who missed the chance to receive them.

The majority $(79.3 \%)$ of the respondents were of the opinion that LLINs can help to prevent malaria and with regards to the acceptability of LLINs; $91.3 \%$ of the respondents liked using mosquito nets against malaria. This is in line with the WHO [18] and Lengeler [1], who stated that on control measures to reduce malaria, the most promising measure is the use of ITNs and curtains. These two factors actually contributed to the high and appropriate use of LLINs among owners as $93.0 \%$ of respondents were using their bed nets to sleep under. These findings are not in conformity with those of Pulford et al. [6] who observed that between $15-50 \%$ of available nets were unused in Nigeria. Also the level of education of the respondents played a significant role in the usage of LLINs because most of the respondents were actually adults with at least a secondary school level of education.

Some studies hold that, mosquito net ownership in itself is not synonymous with utilization $[6,19]$. This is not the case with the findings in this study as most of the respondents that had nets were using them. However, out of those that were using LLINs, $96.7 \%$ were actually using them to sleep under while $3.4 \%$ of the respondents were using them as football nets, nursing seeds, and 
wall cover, harvest bean or to catch fish. This high usage of LLINs among respondents in this locality may be due to public health awareness of the dangers of malaria attack in the studied population and the emphasis on the importance of LLINs to reduce the prevalence of malaria during antenatal and postnatal clinics.

The meaning of effective use varied between respondents. However, whatever the meaning, the important thing is that the LLINs were being used and this offers a degree of protection against the female Anopheles mosquito biting because they bite mostly in the night.

The age of LLINs, how often it has been washed and its general condition is one of the main cardinal factors in the effectiveness of LLINs. This is because even if the LLINs are fitted correctly over the bed within the right hour, old ones with many holes greater than $10 \mathrm{~cm}$ and in which the deltamethrin or permethrin chemical has been washed out will not offer the protection that it is supposed to do since mosquitoes can land on it and bite through the fine holes or go directly into the net. Bachou et al. [5] estimated that LLINs offer greater than $70 \%$ protection compared with no nets. The findings of this study showed that most of the respondents had nets that were in good conditions, and also most of them had not washed their LLINs. All these factors contributed to the effectiveness of their LLINs in the prevention of mosquito bites and the low prevalence of malaria. This roughly supports the report of Yakob and Guiyun [20], which stated that mathematical modeling, has suggested that disease transmission may be exacerbated after bed nets have lost their insecticidal properties under certain circumstances. In this case, since the protection of the LLINs was high, the prevalence of malaria was low.

Some of the respondents in the study said that using LLINs daily was boring and most of the time they were tired and lazy to use them. Others claimed that they did not like using nets because of the bad smell, boring routine exercise of putting them up and down, and the feeling of suffocation. Heat was one of the main problems respondents encountered that could make them not to use LLINs at times, as up to $52.2 \%$ of the respondents said the nets usually gave them heat. This is in line with the reports of Pulford et al. [6].

Education of individuals as well as communities plays a fundamental role in the control/eradication of malaria. From the present study, a strong factor that has reduced malaria cases among the children and pregnant women is education on how to prevent malaria at the primary health care level. This has widened the scope of activities of respondents on keeping the environment clean, clearing bushes around, avoiding standing water in containers, wearing protective clothing and taking antimalarial drugs.

\section{Conclusions}

Pregnant women and guardians need to be sensitized to purchase ordinary bed nets and take them for impregnation at the CTUs if they do not have any. CRAs activities also need to be updated to involve hanging of nets in homes and helping household members to overcome social and personal barriers in the use of bed nets.

\section{Abbreviations \\ ANC: antenatal care clinic; LLINs: long-lasting insecticide-treated bed nets; ITNs: insecticide-treated nets.}

\section{Authors' contributions}

HNN and SV conceived and designed the study. SV analyzed the data and wrote the manuscript. HNN supervised, reviewed and provided inputs to the manuscript. Both authors read and approved the final manuscript.

\section{Author details}

${ }^{1}$ Department of Biological Sciences, University of Bamenda, North West Region, Bambili, Bamenda, Cameroon. ${ }^{2}$ Department of Public Health, Faculty of Health Sciences, Bamenda University of Science and Technology, North West Region, Bambili, Bamenda, Cameroon.

\section{Acknowledgements}

We are grateful to all the pregnant women and the guardians of children who gave their consent to participate in the study. Our special thanks go to the general supervisors, quarter heads and laboratory technicians of PMI Nkwen and Bambui district hospital for their cooperation and contribution.

\section{Competing interests}

The authors declare that they have no competing interests.

\section{Availability of data and materials}

The datasets supporting the conclusions of this article are available in the institutional University of Science and Technology, Cameroon Repository and will be made easily available on request, when required.

\section{Consent for publication}

There are no case presentations that require disclosure of respondents confidential data/information in this study.

\section{Ethics approval and consent to participate}

This study was carried out with the approval of the Ethical Review Committee on Health Research, regional delegation of health for the North West Region, Cameroon. Informed written consent was obtained from each study participant. Each participant was free to withdraw written consent was obtained from each study participant. Each participant was free to withdraw consent at any time. All personal and medical information of the participants was treated with strict confidentiality.

\section{Funding}

Funding was partly from the researchers individual salaries and partly from the Allowances for Research Modernization from the Ministry of Higher Education in Cameroon.

Received: 9 April 2016 Accepted: 4 July 2016

Published online: 26 July 2016 


\section{References}

1. Lengeler C. Insecticide-treated bed nets and curtains for preventing malaria. Cochrane Database Syst Rev. 2004;2:CD000363.

2. WHO. World Malaria Report 2015, Geneva: World Health Organization; 2015.

3. Egbuche CM, Eneanya Cl, Aribodo DN, Eneaya OA, Ogbuagu CN, EzugboNwobi IK. Malaria prevalence and use of insecticide-treated net among communities in Aguleri, Anambra State, Nigeria. Bioscientist. 2013;1:60-6.

4. WHO.: Malaria Fact Sheet No 94. Geneva: World Health Organization; 2014

5. Bachou H, Tylleskär T, Kaddu-Mulindwa DH, Tumwine JK. Bacteraemia among severely malnourished children infected and uninfected with the human immunodeficiency virus-1 in Kampala, Uganda. BMC Infect Dis. 2006;6:160.

6. Pulford J, Hetzel MW, Bryant M, Siba PM, Mueller I. Reported reasons for not using a mosquito net when one is available: a review of the published literature. Malar J. 2011;10:83.

7. Keating J, Hutchinson P, Miller JM, Bennett A, Larsen DA, Hamainza B, et al. A quasi-experimental evaluation of an interpersonal communication intervention to increase insecticide-treated net use among children in Zambia. Malar J. 2012;11:313.

8. Cheesbrough M. District Laboratory practice in tropical countries. 2nd ed. Cambridge: Part 2, Cambridge University Press; 2006. p. 605.

9. Sohail M, Shakeel S, Kumari S, Bharti A, Zahid F, Shadab A, et al. Prevalence of malaria infection and risk factors associated with anaemia among pregnant women in semiurban community of Hazaribag, Jharkhand, India. Biomed Res Int 2015:2015:740512.

10. Leke RGF, Bigoga JD, Zhou J, Fouda GG, Leke RJI, Tchinda V. Longitudinal studies of Plasmodium falciparum malaria in pregnant women living in a rural Cameroonian village with high perennial transmission. Am J Trop Med Hyg. 2010;83:996-1004
11. Mbu RE, Takang WA, Fouedjio HJ, Fouelifack FY, Tumasang FN, Tonye R. Clinical malaria among pregnant women on combined insecticidetreated nets (ITNs) and intermittent preventive treatment (IPTp) with sulphadoxine-pyrimethamine in Yaounde, Cameroon. BMC Womens Health. 2014;14:68.

12. Ndo C, Menze-Djantio B, Antonio-Nkondjio C. Awareness, attitudes and prevention of malaria in the cities of Douala and Yaoundé (Cameroon). Parasit Vectors. 2011;4:181

13. Anchang-Kimbi JK, Nkweti VN, Ntonifor HN, Apinjoh TO, Tata RB, Chi HF, et al. Plasmodium falciparum parasitaemia and malaria among pregnant women at first clinic visit in the mount Cameroon area. BMC Infect Dis. 2015;15:439.

14. Keiser J, Utzinger J, de Caldas Castro M, Smith TA, Tanner M, Singer BH. Urbanization in sub-Saharan Africa and Implication for malaria control. Am J Trop Med Hyg. 2004;71:118-27.

15. WHO. MDG 6: combat HIV/AIDS, malaria and other diseases. Geneva: World Health Organization; 2011.

16. Egrot $M$, Houngnihin R, Baxerres C, Damien G, Djènontin A, Chandre $F$, et al. Reports of long-lasting insecticidal bed nets catching on fire: a threat to bed net users and to successful malaria control? Malar J. 2014;13:247.

17. Kimbi HK, Nkesa SB, Ndamukong-Nyanga JL, Sumbelel UN, AtashiliJ, Atanga MBS. Socio-demographic factors influencing the ownership and utilization of insecticide-treated bed nets among malaria vulnerable groups in the Buea Health District, Cameroon. BMC Res Notes. 2015;7:624.

18. WHO. A global strategy for malaria controls. Geneva: World Health Organization; 1993.

19. The Guardian: Mosquito nets can't conquer malaria. August 2010.

20. Yakob L, Yan G. Modelling the effects of integrating larval habitat source reduction and insecticide-treated nets for malaria control. PLoS One. 2009;: $: 6921$

\section{Submit your next manuscript to BioMed Central and we will help you at every step:}

- We accept pre-submission inquiries

- Our selector tool helps you to find the most relevant journal

- We provide round the clock customer support

- Convenient online submission

- Thorough peer review

- Inclusion in PubMed and all major indexing services

- Maximum visibility for your research

Submit your manuscript at www.biomedcentral.com/submit
O Biomed Central 